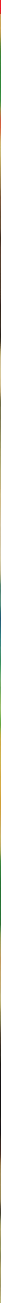

\title{
Archived answers
}

\section{The switch to electronic medical records opens up a potential wealth of data for researchers, if major obstacles can be overcome, reports Katharine Gammon.}

n May 1999, the US Food and Drug Administration (FDA) approved a promising new drug. Earlier clinical trials of 5,000 patients had convinced regulators that the drug was a safe and effective means of reducing pain and inflammation in arthritis. Produced by the pharmaceutical giant Merck, the new medication became an instant hit and nearly 80 million people worldwide had taken this wonder pill by 2004 .

During that same period, however, evidence emerged that the painkiller, known as Vioxx (rofecoxib), significantly raised the risk of heart attacks. By the time Merck pulled the drug from the market in September 2004, "an estimated 88,000 to 140,000 excess cases of serious coronary heart disease probably occurred in the USA", according to a study led by an FDA scientist and published in the Lancet in 2005 (ref. 1). The drug may have caused tens of thousands of deaths in the United States alone, the researchers estimated.

The digital revolution - and the switch from paper to electronic medical records might prevent many of these kinds of deaths in the future. In a $2007 \mathrm{study}^{2}$, researchers from the Children's Hospital Informatics Program (CHIP) in Boston, Massachusetts, attempted to see how quickly they could have spotted a problem with Vioxx using digital records. By homing in on two major hospitals, the researchers found an $18 \%$ jump in heart attack cases within eight months of Vioxx being introduced, which could have provided an early warning about the drug, says Isaac Kohane, an author of the study and director of CHIP. The excess cardiac cases disappeared when Vioxx was pulled from the market.

The Vioxx incident shows one benefit that could emerge when health systems dump old-fashioned paper medical charts in favour of electronic records. Some countries, such as the United Kingdom, have already made the transition and the United States is moving in that direction. This trend could have profound implications for how doctors treat patients and how researchers pursue scientific questions that rely on medical data.

"In the long run, the opportunities are huge - actually transformative," says Ashish Jha, assistant professor of health policy at the Harvard School of Public Health in Boston. "When the exchange of health information flows freely between doctors, we can't imagine how huge that's going to be."

But it will take time. The
"The organization and delivery of health care hasn't changed in nearly a hundred years."

- Ashish Jha move towards electronic medical records has proceeded slowly so far, and many practical challenges still lie ahead. When Jha and his colleagues surveyed US physicians in late 2007 and early 2008, they found that just $4 \%$ have a full electronic records system, and only $13 \%$ have even a basic digital system ${ }^{3}$. "The organization and delivery of health care hasn't changed in nearly a hundred years," says Jha.

That may soon change. The stimulus package that recently passed in US Congress contains US $\$ 19$ billion to promote the switch to digital health records, mostly through economic 
incentives for doctors and hospitals to make the transition. The lofty goal is to have all health records stored and accessed electronically by 2014 .

President Barack Obama has promoted this policy as a way to control soaring health-care costs. An estimated $\$ 81$ billion could be saved annually if $90 \%$ of doctors used electronic systems - by reducing redundant care, speeding up patient treatment and stemming mistakes and handwriting foul-ups. And the switch could cut more than just costs. By reducing the number of medication errors, electronic systems can save lives.

\section{Digging for data}

Proponents of electronic medical records say that researchers could be among the chief beneficiaries of the conversion to digital health data. The fundamental argument for using electronic records in scientific studies is that most doctors don't see enough patients to recognize patterns. Only on the level of hundreds of thousands or millions of records do connections become apparent. What's more, research can be slow when it comes to recruiting patients, evaluating them and checking in over a long period of time, but all that information is available just a few clicks into an electronic record.

But critics argue that current health files are too unstructured, too unreliable and that algorithms for sifting doctors' notes are not yet available.

There's also the question of the type of information in the record. "Depending on what you want to study, that may or may not be consistently documented," says Samuel Skootsky, medical director of the Medical Group and Faculty Practice Group at the University of California, Los Angeles.

Kohane counters that there is enough valuable information in records to justify mining them. What's more, the gigantic amount of patient data available means researchers can choose the individuals to study. For example, investigators looking at asthma could pluck out true asthmatics from people who wheeze owing to other factors, he says.

For research purposes, he says, observations in medical records more accurately reflect clinical practice. "If you study a drug and you look at its effect on a control group, that's a very pure study," says Kohane. "But everyone taking that drug might be also taking two other drugs related to their condition", which could alter the effects of the medication being studied. That information is not always available

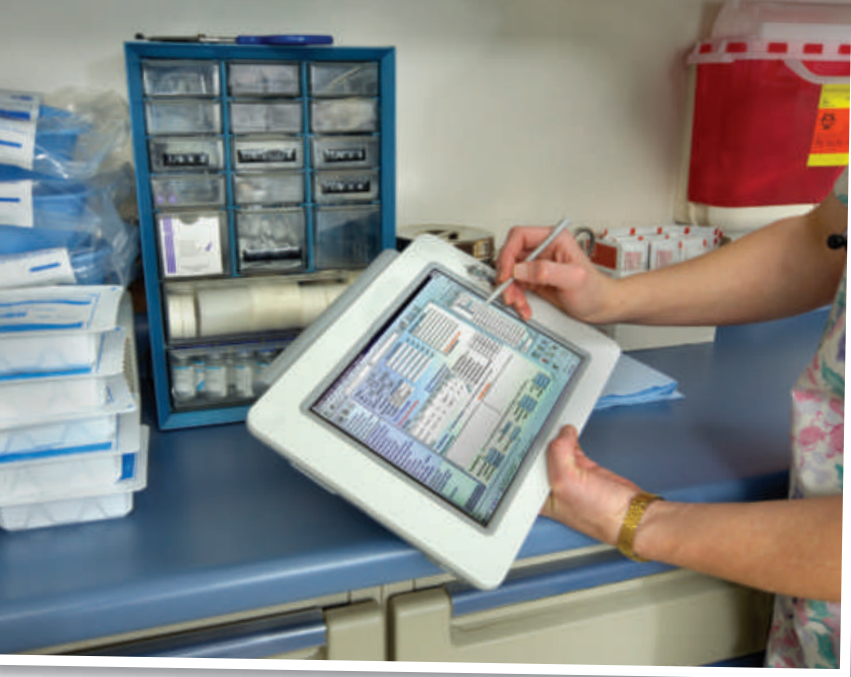

alth records can save lives and money, but security is a concern.

Cedars-Sinai hasn't been the only institution to face a rebellion. In the United States, electronic medical records spark such fierce resistance in part because those who benefit most from these systems - the insurance companies and billing department — aren't the same as the people who have to pay for the new software and training. For a medium-sized hospital, these systems can cost tens of millions of dollars just for software - with no guarantee of a return on investment for the hospital. As nearly

to researchers conducting trials, but electronic medical records might help sort out the multidrug effects.

In a test of that potential, Kohane's group is using health records to look at other drugs on the market. The team is searching for effects that might have been missed in earlier tests of the medications.

Even more power might emerge by combining clinical records with a genetics database. Michael Swiernik, director of medical informatics at the University of California, Los Angeles, says that the combination would allow researchers to find the right people for a study in a speedy, cost-effective way.

Other research affirms that

"What you want to
study may or may
not be consistently
documented."
- Samuel Skootsky
half the doctors in the United States practise either alone or with one partner, the cost is extreme: $\$ 32,000$ per physician for installation and $\$ 1,500$ per month for running the system, according to estimates.

When a hospital or doctor goes out to buy a system, they face another challenge: there are hundreds of vendors who each want to sell a different, proprietary product, many of which will not survive. In addition, medical records systems often have problems sharing data with each other. Taken together, these issues "make doctors and hospitals very nervous to purchase the technology", says Jha.

That apprehension can extend to using the systems as well: studies show that doctors' it is possible to dig into electronic records for useful clinical data. A study released in February showed that data captured in electronic records could yield the same results as data from clinical trials when evaluating the efficacy of a drug ${ }^{4}$. The data came from Britain's general practice research database, which holds about $10 \%$ of the records of general practitioners mostly relating to demographics and pharmaceutical usage. The problem is that these UK databases are small, incomplete and are not yet linked together.

Before researchers can truly tap digital records, the databases first have to be created and filled. That will not happen if the primary users reject them, as happened at the CedarsSinai Medical Center in Los Angeles in 2002. After undergoing months of training with a new electronic records and communications system, doctors complained that it was too cumbersome. One day, the staff revolted, ending the hospital's \$34-million effort to convert to an electronic system after just three months. efficiency actually drops 10-20\% during the first year of using an electronic system.

Then there's the thorny issue of privacy. Moving electronic patient information around between doctors, pharmacies, laboratories and billing departments means many people have access to sensitive information. That raises the risk of a large security breach. Medical-record information sells for more on the black market than credit-card information, because it is more identifying, says Swiernik.

"Security is only as good as its weakest link, and the likelihood of weak links is really pretty high," says Lee Tien, a lawyer at the Electronic Frontier Foundation, a digital-rights publicinterest group based in San Francisco, California. He is particularly concerned about data security because the transition to electronic health records is happening so quickly. "If you're not hard-wiring privacy and security into the DNA of the infrastructure, there will be unfortunate data security breaches."

Despite these challenges, some institutions 


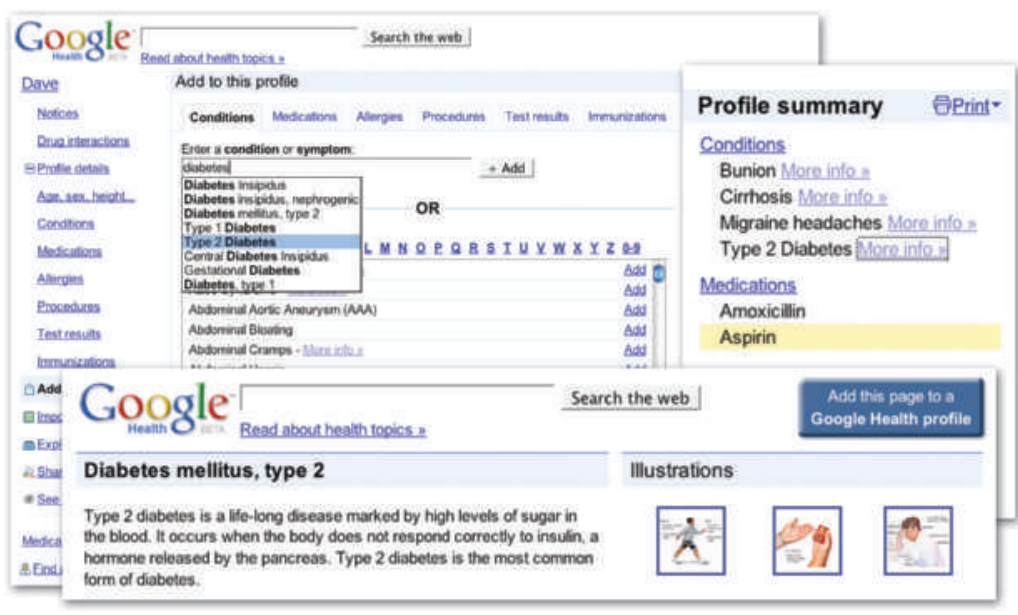

Consumers are storing their medical data online with systems such as Google Health.

by patients, not institutions. Models of personal health records already exist in systems such as Google Health, Microsoft's HealthVault and Indivo, which was built by CHIP researchers. They can all store patient data imported from doctors' offices or laboratories, and give control of the data to the patient. When it comes to scientific research, Kohane says personal records could connect patients directly with researchers without hospitals or physicians as medical records, and nearly half of them use the online interface, where they can view part of their records.

But those systems have yet to benefit researchers substantially. Because of privacy regulations, Kaiser Permanente, based in Oakland, California, limits access to its clinical records mostly to researchers within the organization. The VA has also been slow in research. "The VA has had electronic data for 10 years now, but only a small amount of stuff is available for digital clinical research," says Jha, adding that the technology and the level of comfort with anonymized data sharing hasn't yet caught up to the amount of data available.

The largest electronic medical-records system in the world is Britain's National Health Service (NHS), serving more than 60 million people. But the digital data amassed by UK doctors is not yet comprehensively linked up. That will change in the next five years, by which time electronic records will connect 30,000 general practitioners and include data on all UK residents.

Despite that push into the digital age, the opportunity for research has been missed, says John Powell, associate professor in epidemiology and public health at the University of Warwick, UK. "There's a massive debate going on right now about consent - whether
"If you're not hardwiring privacy into the DNA of the infrastructure, there will be data security breaches." - Lee Tien criticism. Powell says many of the issues will be resolved this year, when the NHS finalizes its official constitution - a sort of terms-ofservice agreement for using the system. He argues that the system should be used for research. "In return for getting completely free health care for everything, there are certain things you have to sign up for as a resident - and one of those things should be secondary use of your data for the anonymous NHS research, but not for pharmaceutical companies," says Powell.

\section{Security fears}

Britain has faced major data security problems in the past few years, so people are understandably concerned about the safety of their medical information. But there are rumblings of change: the creation in February of the National Institute for Health Research, a framework agency within the NHS, should make it easier for anonymized data to move within the system.

Other European nations haven't decided what to do with their records, either. In Germany, doctors have local electronic health records, but no way to connect them to a larger system. France has a national system to hold and patients are opting in or opting out of a research system. Doctors for the most part are arguing that people have to give consent to opt in, civil servants in the Department of Health are saying they have to opt out. From the research point of view, we want to have maximum people and maximum data in." General practitioners tend to be wary of sharing patient data, even if they are de-identified.

That concern grew recently, when some officials suggested that the NHS could sell data to pharmaceutical companies to raise funds - a proposal that prompted strong access electronic medical records, although clinicians can view only the part of the record that is relevant to them.

Some researchers see an alternative to large, expensive systems in something closer to Apple's iPhone, with its panoply of available applications. Kohane says that the best system would be a platform available with different inter-operable, substitutable programs.

If open-source platform systems aren't built because of the nature of the vendors, Kohane sees a future in the expansion of personal health records - computerized records maintained intermediaries - even offering the anonymity required by most review boards.

Such systems could fix a major problem in medical research. Right now, people who participate in studies do so anonymously, and the results are not usually transmitted back to them. Kohane and his colleagues are testing an approach later this year that will allow for more communication in one Boston clinic with 5,000 patients, and they are adding more clinics in 2010. Patients will sign up at their health-care institution and upload clinical or genomic information to an encrypted personal health record to be shared with researchers. Then patients could choose to 'tune in' to a certain type of announcement regarding their records.

If results came out that matched their genomic profile, patients would get a message about that work. They could also sign up for getting news on particular conditions, such as cancer or diabetes. And researchers could communicate with patients without knowing their identities, allowing an ongoing relationship. "The current state of research is of self-inflicted mutual ignorance," says Kohane. "Patients are committing to not knowing more about the study, and researchers can't find out more information about the patients."

All that could change if health systems can get past the substantial hurdles. In a world where information is at our fingertips, and even the US president has his thumbs on a BlackBerry, electronic medical records are coming, along with the research opportunities they hold. For many scientists, that transition is just what the doctor ordered.

Katharine Gammon is a freelance writer based in Santa Monica, California.

1. Graham, D. J. et al. Lancet 365, 475-481 (2005)

2. Brownstein, J. S., Sordo, M., Kohane, I. S. \& Mandl, K. D. PLOSONE 2, e840 (2007).

3. DesRoches, C. M. et al. N. Engl. J. Med. 359, 50-60 (2008).

4. Tannen, R. L., Weiner, M. G. \& Xie, D. Br. Med. J. 338, b81

(2009).

See Editorial, page 259. 